\title{
A Distributional and Sensorimotor Analysis of Noun and Verb Fluency
}

\author{
Mengyang Qiu \\ University at Buffalo \\ Brendan T. Johns \\ McGill University
}

Words: 5,582

Corresponding Address

Brendan Johns

Department of Psychology, McGill University

2001 McGill College Avenue

Montreal, Quebec, Canada

H3A 1 G1

Acknowledgements: This research was supported by Natural Science and Engineering Research Council of Canada (NSERC) Discovery Grant RGPIN-2020-04727 to BTJ. 


\begin{abstract}
Category verbal fluency tasks, where participants are asked to produce words according to a semantic category, are typically noun-based (e.g., animals). While insights about the integrity and retrieval of semantic knowledge have been obtained by analyzing the ordinal variances of word production in these noun-based fluency tasks, focusing exclusively on noun categories ignores the semantic knowledge contributed by other semantic/grammatical categories, especially verbs. To better understand the representational differences of nouns and verbs within the mental lexicon, the current study conducted and contrasted different noun- and verb-based fluency tasks. By analyzing the use of different lexical information sources, including word frequency, and context and order similarity derived from a computational model of lexical semantics (BEAGLE; Jones \& Mewhort, 2007), and the use of perceptual information derived from the recently released sensorimotor norms (Lynott, Connell, Brysbaert, Brand, \& Carney, 2020), it was found that these information sources consistently distinguished noun and verb retrieval, signaling the underlying distributional and sensorimotor representational differences for these two semantic/grammatical categories. The results demonstrate the essential and integral role that distributional and grounded/embodied models play in understanding language and cognition, and highlight the usefulness of verbal fluency tasks in exploring theoretical questions in memory and psycholinguistics.
\end{abstract}

Keywords: verbal fluency; semantic memory; distributional semantics; embodied cognition 


\section{A Distributional and Sensorimotor Analysis of Noun and Verb Fluency}

Verbal fluency is a widely used cognitive task in both clinical and research settings. In this task, participants are asked to produce as many unique words as possible from a given criterion within a certain amount of time (e.g., one minute). Letter/phonemic fluency (producing words starting with a certain letter/sound, e.g., $F, A$, and $S$ ) and category/semantic fluency (producing words within a semantic category; e.g., animals, vegetables, or pieces of furniture) are two of the main verbal fluency tasks used. These tasks are used to assess language processing, executive functioning, and semantic memory across a variety of populations, including bilinguals (Patra, Bose, \& Marinis, 2020; Taler, Johns, Young, Sheppard, \& Jones, 2013), healthy aging (Pakhomov, Eberly, \& Knopman, 2016; Taler, Johns, \& Jones, in press), and people with mild cognitive impairment (MCI), Alzheimer's disease (AD) and other neurocognitive deficits (Taler, Monetta, Sheppard, \& Ohman, 2020; Taler \& Phillips, 2008; Thiele, Quinting, \& Stenneken, 2016).

Category fluency, in particular, has received attention because performance in this task provides valuable insight into the integrity of semantic memory and patterns of semantic knowledge retrieval. One well-established measure of category fluency performance is the cluster-and-switch approach, which analyzes the size and coherence of semantic clusters (i.e., word subcategories, for example, pet, farm animals, and African animals for the animal category) and the number of switches between subcategories (Troyer, Moscovitch, \& Winocur, 1997; Troyer, Moscovitch, Winocur, Leach, \& Freedman, 1998). Smaller and less coherent semantic clusters are a good indicator of the underlying degradation of semantic knowledge in people with MCI and AD (Murphy, Rich, \& Troyer, 2006).

However, there is one major drawback of the original cluster-and-switch approach, which is that semantic clusters are hand-coded, meaning that this approach is timeconsuming and invites issues of inter-rater reliability. An alternative methodology to hand-coded ratings is the use of corpus-driven cognitive models to assess the semantic relationship between words (e.g., Hills, Jones, \& Todd, 2012; Taler, et al., 2013). There are now a wide variety of models that can exploit the statistical structure of word occurrence within natural language (entitled distributional models of language; e.g., 
Landauer \& Dumais, 1997) as well as various linguistic corpora available to train them on (e.g., Wikipedia articles, books, and film/television subtitles; Johns, Jones, \& Mewhort, 2019). Thus, it is now possible to measure word relations more objectively. The use of these model types have been successfully applied to automatically measure verbal fluency performance in order to better understand the underlying cognitive mechanisms of memory search (e.g., Abbott, Austerweil, \& Griffiths, 2015; Avery \& Jones, 2018; Hills, Jones, \& Todd, 2012; Johns et al., 2018; Taler et al., 2013, in press).

The natural language environment is an essential source for the acquisition and representation of semantic knowledge. Distributional models of semantics, or semantic space models, learn word meanings from statistical regularities within the natural language environment and represent them as high-dimensional vectors (for a review, see Günther, Rinaldi, \& Marelli, 2019 and Jones, Willits, \& Dennis, 2015). The intuition behind these models is that the meaning of a word is related to its usage in context, as stated in Firth (1957), "You shall know a word by the company it keeps". The resulting representation from a distributional model allows for the similarity of words to be computed, providing insight into the connection between people's internalized representation of word meanings to the statistical pattern of word occurrence within large textbases (presumably derived from sources that are coherent to the linguistic experience of people; Johns \& Jamieson, 2019).

Hills et al. (2012; see also Hills, Todd, \& Jones, 2015) proposed a foraging model with an exploration-exploitation mechanism to explain animal fluency data. Their model operated on word similarities derived from a distributional model (BEAGLE; Jones \& Mewhort, 2007). The modeling results showed that participants used local similarity (i.e., distributional similarity to the last word produced) to organize word production, and word frequency to switch to a new subcategory when no proximal word was found in semantic space. Although the mechanism is similar to the cluster-and-switch approach, the two differ in important ways. For example, in addition to the fully automated nature of the memory foraging model, the model is able to incorporate multiple types of linguistic information, rather than just subcategory membership, to infer the retrieval patterns underlying the sequence of words produced.

Johns et al. (2018) used a model derived from the foraging model of Hills, et al. to examine the longitudinal changes in memory search pathway underlying animal fluency in 
pre-MCI patients and healthy controls over a two-year period. Johns et al. (2018) found that as pre-MCI patients progressed to MCI, they began to generate words with higher frequency. Additionally, these words also became closer to each other in semantic space. These results demonstrate that changes in semantic knowledge may occur before cognitive dysfunction is formally diagnosed and shows the unique advantages that computational modeling provides to the analysis of verbal fluency data.

Most category fluency studies have focused on noun-based categories, typically animals. Clearly, the use of such a restricted number of categories only enables an examination of a limited amount of semantic knowledge. Although several studies in the neuropsychological literature have proposed verb fluency (i.e., generating actions that people do) as an alternative diagnostic tool (e.g., Östberg, Fernaeus, Hellström, Bogdanović, \& Wahlund, 2005; Piatt, Fields, Paolo, Koller, \& Tröster, 1999; for a review, see Beber \& Chaves, 2014), the ordinal variances of verb production have been largely unexplored. Cross-linguistically, verbs have been found to be semantically, syntactically, and morphologically distinct from nouns (Vigliocco, Vinson, Druks, Barber, \& Cappa, 2011). Most crucially, verbs are semantically more complex than nouns. While nouns typically refer to objects or entities, verbs describe actions, or more generally, events, in which there is a relationship between one or more entities (i.e., argument structure; Koenig, 2016). This difference in semantic content and complexity is also reflected in the differences in cognitive demand and brain activation of noun and verb processing (Moseley \& Pulvermüller, 2014; Vigliocco, et al., 2011). Therefore, examining the memory search patterns contrasting noun and verb production may yield additional understanding about the organization and retrieval of semantic knowledge.

The goal of the current study is to use a combination of computational modeling and recently released normed sensorimotor information (Lynott, Connell, Brysbaert, Brand, \& Carney, 2020) to better understand the representational differences of nouns and verbs. This will be accomplished through the analysis of two categorical verbal fluency experiments, where different noun- and verb-based fluency tasks were conducted and contrasted. Through analysis of this data, it will be shown that nouns and verbs have different representational properties that can be objectively assessed with modern psycholinguistic tools, a new trend in the cognitive and language sciences. The 
information sources examined are word frequency, context (semantic) and order (syntactic) distributional similarity, and sensorimotor similarity. It will be shown that these information sources have reliably different properties across nouns and verb fluency.

Computational modeling of verbal fluency has typically focused on contrasting different theoretical notions of memory search, for example the foraging proposals of Hills et al. (2012) and the random search model of Abbott et al. (2015; see Jones, Hills, \& Todd, 2015 and Avery \& Jones, 2018 for a comparison and discussion of these two models). The goal of this article is not to propose a new model of verbal fluency, but instead to explore the underlying representational differences that govern verbs and nouns. Both the foraging model of Hills, et al. and the random search model of Abbott, et al. depend on the strength of connection between words to account for verbal fluency performance. In the standard version of these models, the probability of switching from word $x$ to word $y$ is dependent on two types of information: 1) the word frequency of word $y$ (entitled the bias term), and 2) the semantic similarity between the words (derived from BEAGLE for the foraging model, and from free association norms for the random search model). The success of these models demonstrates that the pairwise semantic similarity, combined with word frequency, provide a parsimonious accounting of verbal fluency data.

However, the sole use of lexical information sources ignores current trends in the cognitive sciences, where the influence of perceptually grounded and embodied information on language processing has been emphasized (e.g., Barsalou, 1999, 2008; Connell \& Lynott, 2012, 2014; Heard, Madan, Protzner, \& Pexman, 2019; Johns \& Jones, 2012; Louwerse, 2008, 2011; Pexman, in press; Siakaluk, et al., 2008). Indeed, Johns, et al. (2018) demonstrated that integrating grounded information into a model derived from the foraging model of Hills, et al. increased the fit of the model on animal fluency data, suggesting that this type of information is actively used in verbal fluency. Thus, in addition to lexical information derived from corpusbased models, the differences in the usage of sensorimotor similarity in verb and noun fluency will also be examined in the following analysis.

The combined use of lexical and grounded/embodied information to assess language processing (e.g., Andrews, Vigliocco, \& Vinson, 2009; Johns \& Jones, 2012; Riordan \& Jones, 2011) is an important trend in computational cognitive modeling, reinforced by many large-scale data collection projects assessing the interaction of language and grounded/embodied 
information (e.g., Bennett, Burnett, Siakaluk, \& Pexman, 2011; Lynott \& Connell, 2009, 2013; Lynott, et al., 2020; McRae, Cree, Seidenberg, \& McNorgan, 2005; Pexman, Muraki, Sidhu, Siakaluk, \& Yap, 2019; Tillotson, Siakaluk, \& Pexman, 2008; Sidhu, Kwan, Pexman, \& Siakaluk, 2014). The use of both information types acknowledges that the lexical environment and the perceptual environment are key to explaining language processing. The results of this article will demonstrate that the continued interaction and integration of theories of language with theories of perception provides an important pathway to the development of new cognitive theory.

The outline of this article is as follows. The first section will describe the cognitive models and sensorimotor norms used to analyze the verbal fluency data collected here.

Following this, the first experiment will contrast a noun-based category fluency task with a verbbased task. The second experiment will demonstrate that the results of the first experiment were not due to the particular characteristics of the categories chosen, and generalize to very different noun- and verb-based categories. Overall, this article will demonstrate that there are consistent, strong, and objectively interpretable differences in the underlying representations of verbs and nouns within the mental lexicon. Additionally, this article will point to the usefulness of verbal fluency as a behavioral task of general importance within theoretical psycholinguistics.

\section{Analysis Outline}

The different information sources that will be used to analyze the differences in noun and verb fluency will be described. As stated previously, there are four types of information that will be used: 1) word frequency, 2) distributional context similarity, 3) distributional order similarity, and 4) sensorimotor similarity. Each will be described in turn.

Word frequency and corpus. Word frequency (WF) is calculated by counting the

number of times a word occurs across a corpus of natural language. WF is a standard measure in psycholinguistics, and has ubiquitous effects across language and memory processing (see Brysbaert, Mandera, \& Keuleers, 2018 for a recent review). Here WF was calculated for every word produced in the following verbal fluency experiments.

The corpus used to measure WF, as well as to train the below described model, is a corpus derived from over 25,000 fiction and non-fiction books. This corpus has been used to explore lexical decision and naming times (Johns, Dye, \& Jones, 2020), familiarity and lexical 
semantic data (Johns \& Jamieson, 2019), personal name distributional properties (Johns \& Dye, 2019), sentence processing (Johns, Jamieson, Crump, Jones, \& Mewhort, 2020), as well as the analysis of large-scale category fluency data (Taler, Johns, \& Jones, in press). In total, this corpus contains approximately two billion words, a sizeable amount of language. The advantage of using books over other types of corpora is that they contain a relatively greater number of low frequency words than other corpora, enabling a more complete analysis of the language space (Johns, Dye, \& Jones, 2020).

The BEAGLE Model. The distributional semantic model that will be used here is BEAGLE (Jones \& Mewhort, 2007), a standard distributional model of semantics, inspired by the classic TODAM model of episodic memory (Murdock, 1982, 1995). For the purposes of this article, the important aspect of BEAGLE is that it encodes both context and order information. Typical distributional models, such as Latent Semantic Analysis (Landauer \& Dumais, 1997) or neural embedding models such as word2vec (Mikolov, et al., 2013) only learn context information (or the co-occurrence patterns of words). Jones and Mewhort (2007) demonstrated that the dual information types learned by the model allow for different aspects of nouns and verbs to be learned, and so the use of this model is particularly well suited to the goals of this article.

BEAGLE works by "reading" a text corpus a sentence at a time and through this experience, encode each word's context and order meaning into a set of corresponding vectors. In BEAGLE, there are two types of vectors: environmental and memory vectors. Environmental vectors are static (meaning they do not change across learning), and are assumed to represent the perceptual properties of a word. There are two of memory vectors - context and order - and both are dynamic, in that they accumulate new information as words are used in language. The context vector accumulates sentential co-occurrence information. The order vector accumulates n-gram information about how a word is used within a sentence.

All vectors have a dimensionality of size $N$ (set at 2,048 for this article). Environment vectors are random Gaussian vectors with a mean of 0 and a standard deviation of $\sqrt{1 / N}$. For word $i$, the context vector, $\mathbf{c}_{i}$, for that word is updated by summing the words that occur with that word in a set window size ${ }^{1}$. After training, the context vector encodes the words that a target

\footnotetext{
${ }^{1}$ Window size was set at 5 words. The window does not include the target word, but instead the words surrounding the target.
} 
word co-occurs with. When training context vectors, a stop list (taken from Landauer \& Dumais, 1997 ) is used to remove very high frequency function words.

The order vector is updated by encoding linear n-grams of the words that surround a target word in a sentence. To encode n-gram information, directional circular convolution (Jones \& Mewhort, 2007; Plate, 1995) is used. Circular convolution is a function that takes in two vectors and constructs a new, unique vector that represents the association between the two. By building up the associations of $n$-grams within a set window, the order vector provides information about how a word is used within a sentence in relation to other words.

Overall, a word's context vector represents pure co-occurrence information whereas order information encodes a simplified representation of syntactic relations. Despite its simplicity, BEAGLE explains a broad range of semantic and language behavior (e.g., Johns, et al., 2018; Hare, Jones, Thomson, Kelley, \& McRae, 2009; Johns \& Jamieson, 2019; Johns \& Jones, 2015; Johns, Mewhort, \& Jones, 2019; Johns, Jamieson, Crump, Jones, \& Mewhort, 2020; Jones, Kintsch, \& Mewhort, 2006; Jones \& Mewhort, 2007; Taler, et al., in press). Both types context and order similarity will be used in the below analysis.

Sensorimotor representation. Sensorimotor similarity is calculated by using the Lancaster Sensorimotor Norms, a crowdsourced database that includes measures of sensorimotor strength for nearly 40,000 English words (Lynott, et al., 2020). Specifically, words are normed on a $0-5$ scale across six perceptual modalities (i.e., to what extent a word is experienced by touching/hearing/smelling/tasting/vision/interoception) and five action effectors (i.e., to what extent a word is experienced by the action of the foot/leg, hand/arm, mouth/throat, head excluding mouth, and torso), resulting in an 11-dimension sensorimotor representation for each word. Lynott et al. (2020) demonstrated that the combination of both perceptual and action strength measures provided a superior fit for lexical decision performance.

One advantage of using the Lancaster Sensorimotor Norms in the current study over other embodied measures (e.g., body-object interaction ratings; Pexman, et al., 2019) is that the multidimensional representation of Lynott et al. allows for a more comprehensive and finegrained comparison between words. For example, consider the three animal words, zebra, giraffe and squid. These words have the same mean body-object interaction ratings (4 on a 1-7 scale; Pexman, et al., 2019), but the modality/effector-specific representation shows that zebra is similar to giraffe across more dimensions than to squid (see top panels of Figure 1), leading to a 
higher pairwise sensorimotor similarity between zebra and giraffe. Similarly, while the three verbs, push, cuddle and speak, have the same mean relative embodiment ratings (6.19 on a 1-7 scale; Sidhu, et al., 2014), push and cuddle are more similar to each other across most sensorimotor dimensions (see bottom panels of Figure 1).

In addition, nouns and verbs are rated along the same 11 sensorimotor dimensions in the Lancaster Sensorimotor Norms. This makes it possible to directly compare the usage of sensorimotor information across noun and verb-based fluency tasks. The comprehensiveness of the norms also makes it the only sensorimotor measure available that covers most words produced in the fluency tasks in the current study. Over $92 \%$ of the words produced in the two noun-based fluency tasks and $98 \%$ of the words in the verb-based fluency tasks are normed in the Lancaster Sensorimotor Norms.

To calculate sensorimotor similarity, the six perceptual modality ratings and five action effector ratings were put into a vector. The sensorimotor similarity between the vector representation of two words is computed with a vector cosine, which returns a value between -1 and 1 . A vector cosine was selected because it is the same similarity metric that BEAGLE utilizes, and so all similarity measures will be on the same scale.

Analysis technique. Previous models of verbal fluency have a used a memory search model, with different information sources being parameterized (e.g., Hills, et al., 2012; Johns, et al., 2018). Here, a different tactic will be used, based off of the recent work of Taler, Johns, and Jones (in press), where instead of fitting a model to the data, the pattern of word frequency and pairwise similarity of words produced in a verbal fluency task were analyzed. Thus, for each participant analyzed here, the average word frequency, pairwise context similarity, pairwise order similarity, and pairwise sensorimotor similarity were calculated for all word's that the subject produced. If systematic differences in these values are found in noun and verb fluency, then it would suggest that there are different underlying representational components of nouns and verbs that emerge in the different information sources used here.

\section{Experiment 1: Animal and Verb Fluency}

As a first attempt to understand the differences between verb and noun verbal fluency, two types of categorical fluency were collected. One was the classic animal fluency task. In the other task, participants were asked to only produce verbs as in previous verb fluency studies (e.g., Östberg, 
et al., 2005).

Participants. Fifty native English speakers (age $M=35.82, S D=10.17$; education $M=$ 14.94, $S D=1.92$ ) were recruited from Amazon Mechanical Turk. Each participant was paid $\$ 1.00$ for their participation. All procedures were approved by the University at Buffalo Institutional Review Board (IRB). Informed consent was obtained prior to the start of the experiment. The use of a mix of older and younger participants was decided in order to enable a more representative sample, given that there are some changes in verbal fluency across the aging spectrum (Taler, et al., in press).

Procedure. The experiment was implemented using the psiTurk framework (Gureckis, et al., 2016). Each participant completed three verbal fluency tasks online. The task instructions were adapted from the Montreal Cognitive Assessment (MoCA; Nasreddine, et al., 2005). Specifically, participants were asked to say as many words as they could think of that conform to a criterion to be displayed on screen in one minute. In addition, they were instructed not to say words that are proper nouns (e.g., Bob or Boston), numbers, or the same words with different suffixes (e.g., love, loves, lover, and loving). The first verbal fluency task was letter fluency (criterion: words that begin with the letter $F$ ), which was a practice trial in order for the participants to be familiar with the task. After that, participants completed an animal fluency (criterion: types of animals) and a verb fluency (criterion: things that people do, actions/verbs) task in randomized order. For each task, their verbal responses were recorded after they hit the 'Record' button on screen. The recording would automatically stop after 1 minute, and then be saved as a .wav file and transferred to a local server. All the collected audio files were first transcribed by the Google Speech-to-Text api $^{2}$. The transcriptions were then manually checked for repetitions, out-of-category words, and homophone spelling errors (e.g., dear to deer in animal fluency). Multiword animal names and verb+preposition phrases were concatenated into a single word (e.g., polar bear to polarbear, tidy up to tidyup), and the corpus used to derive frequency and semantic representations was preprocessed accordingly.

Data availability. Mean and item-level behavioral and model data is available at https://osf.io/cgdr2/.

Behavioral Results. The left panel of Figure 2 displays the number of items produced in

\footnotetext{
${ }^{2}$ https://cloud.google.com/speech-to-text
} 
the animal and verb fluency task. This figure shows that on average, participants produced more animals than verbs, a statistically significant result $[t(49)=4.353, \mathrm{p}<0.001]$. This suggests that participants found producing animals easier than producing verbs. For animal fluency, there were 205 total types produced, while for verb fluency there were 319 types produced, suggesting that the category size of verbs was greater than that of animals, an intuitively correct result.

Modeling Results. Figure 3 displays the average word frequency, and average context, order, and sensorimotor pairwise similarity for the animal and verb fluency tasks. This figure shows that there are large and consistent differences across all information types. A repeated measures ANOVA confirmed that all four differences were highly significant, with verbs having an average higher word frequency $[F(1,49)=580.35, \mathrm{p}<0.001]$, as well as a higher average context similarity $[F(1,49)=35.18, \mathrm{p}<0.001]$, while animals had a higher average order similarity $[F(1,49)=230.45, \mathrm{p}<0.001]$ and sensorimotor similarity $[F(1,49)=382.26, \mathrm{p}<0.001]$.

These findings suggest that verbs tend to cluster more on context similarity, meaning that sequentially produced verbs are used with similar words in context, while the animals tended to cluster more on order similarity, signaling that they are being used in the same manner across sentences. Additionally, the animals produced were higher in sensorimotor similarity, signaling that their representations are likely more perceptually grounded, compared to verbs. Additionally, the verbs produced were of higher overall word frequency.

Discussion. This experiment was a first attempt at determining representational differences between verbs and nouns using verbal fluency in combination with distributional modeling techniques and newly released sensorimotor norms. Four different information sources were selected to determine the difference in noun and verb fluency: 1) word frequency, 2) context similarity, 3 ) order similarity, and 4) sensorimotor similarity. It was found that there were large and systematic differences between nouns and verbs produced for each information source.

However, before commenting on the theoretical significance of this result, there is one large potential confound in experimental design that needs to be addressed: the category size difference between the animal and verb categories. Animals represent a closed category type while verbs represent an open, and rather abstract, category type. It is possible that this category size difference is driving the difference in verbal fluency patterns, and not underlying representational differences between nouns and verbs. In order to test this possibility, the next 
experiment will contrast an open-ended noun category task with a closed verb category. If similar modeling results emerge using these tasks, then it would indicate that there are systematic differences between nouns and verbs in terms of their representation in the mental lexicon.

\section{Experiment 2: Noun and Body-Movement Fluency}

To flip the previous category instructions, here the noun category will be open-ended while the verb category will be closed. To accomplish this, for the noun category, participants were simply asked to produce as many nouns as they could. For verbs, we took inspiration from recent embodied psycholinguistic word norms, namely body-object interaction values (Bennett, Burnett, Siakaluk, \& Pexman, 2011; Pexman, Muraki, Sidhu, Siakaluk, \& Yap, 2019; Tillotson, Siakaluk, \& Pexman, 2008). Specifically, for verb fluency, participants were asked to produce as many words as they could that involve actions that they could produce with their body (this will be referred to as the body movement task). That is, noun fluency will now be abstracted from a particular semantic category, while the verb category will be restricted to a limited subset of possible verbs.

Participants. Fifty-six native English speakers (age $M=35.38, S D=10.02$; education $M$ $=14.89, S D=2.09$ ) were recruited from Amazon Mechanical Turk. Each participant was paid $\$ 1.00$ for their participation. Participants from Experiment 1 were automatically excluded from taking the second experiment using psiTurk. There was no significant difference between the participants in the two experiments in terms of age $(p=0.82)$ and years of education $(p=0.90)$.

Procedure. The procedure was the same as Experiment 1. After the participants finished the letter $F$ fluency task, they completed a noun fluency (criterion: names of any living or nonliving things) and a body-movement fluency (criterion: actions that involve your body movement) task in randomized order.

Behavioral results. The right panel of Figure 2 displays the number of words produced for the two tasks. This panel shows similar results to experiment 1 , where subjects were able to produce more nouns than verbs, with that difference being significant $[t(1,55)=7.91, \mathrm{p}<0.001]$. Compared to the verb condition in Experiment 1, participants had a greater degree of difficulty producing body movement words. Importantly, in this experiment, participants produced 492 word types in the noun fluency condition, while they produced only 232 body movement word types, demonstrating the category size for nouns and verbs is the opposite of what was found in 
Experiment 1. If it is the different category sizes that is driving the different model values found in Experiment 1, then there should be a reversal in the values in this experiment.

Modeling results. Figure 4 displays the average word frequency, and average context, order, and sensorimotor pairwise similarity for the noun and body movement fluency tasks. This figure shows that the model values for the noun and body movement fluency tasks are very similar to the animal and verb fluency values from Experiment 1 . The difference between values was again assessed with a repeated measures ANOVA. The word frequency difference was smaller than in Experiment 1, but was still significant $[F(1,55)=10.069, p=0.002]$. For the other model values, the results were consistent with the noun versus verb difference observed in Experiment 1, with the participants producing more similar words in body movement fluency task $[\mathrm{F}(1,55)=29.235, \mathrm{p}<0.001]$, but the noun fluency words being closer in order space $[F(1,55)=160.252, p<0.001]$ and sensorimotor similarity $[F(1,55)=25.01, p<0.001]$. Overall, even though Experiment 1 used different category labels for noun and verb fluency than Experiment 2 , the model values were completely consistent across the different tasks.

Discussion. Experiment 2 was designed to determine the consistency of the model values found for noun and verb category verbal fluency where the size of the categories was flipped from Experiment 1. Instead of animal and verb category fluency, Experiment 2 used noun and body movement fluency. It was confirmed that the noun category contained more word types than the movement category (similar to how the verb category contained more types than the animal category, meaning that the category sizes were flipped from Experiment 1 to Experiment 2). Additionally, it was found that the model values for the two fluency categories from Experiment 2 were identical to those found in Experiment 1, demonstrating that these values were not due to category size but instead due to an internal consistency in the representational differences between nouns and verbs. The theoretical significance of this result will be discussed in the General Discussion.

\section{General Discussion}

The goal of this article was to use verb and noun verbal fluency data to determine the representational differences of nouns and verbs in the mental lexicon. In Experiment 1, animal fluency was used to generate noun-based data, while verb fluency was used to generate verbbased data. Experiment 2 attempted to solve the potential confound of category size by using an 
open-ended category structure for nouns, where participants were asked to produce as many nouns as possible, while a closed category structure was used for verbs, where participants were asked to produce as many body movement verbs as possible. Consistent model values were found across both experiments, indicating distributional and sensorimotor representational signatures for these two grammatical classes.

The differences found for each psycholinguistic information source will be discussed in turn. For word frequency, it was found in both experiments that the average word frequency of the verbs produced was higher than for the nouns. Taler, et al. (2013) demonstrated that when task difficulty was increased (in this experiment, by using a language switching task where subjects had to switch between using English and French), there was a corresponding shift towards using higher frequency words. Similarly, Johns, et al. (2018) found that patients with MCI produced higher frequency words in category fluency. Given that in both experiments reported here participants produced fewer verbs on average, the use of higher frequency words likely signals a greater difficulty in retrieving verbs, leading to a greater reliance on using frequency compared to nouns.

There were two types of distributional similarity values used - context and order similarity derived from the BEAGLE model of distributional semantics (Jones \& Mewhort, 2007). Context similarity represents word co-occurrence information whereas order information encodes a simplified representation of syntactic relations. It was found that verbs had a higher pairwise context similarity. In contrast, it was found that both noun categories had a higher pairwise order similarity. The difference in order similarity is coherent with the fact that verbs are more syntactically complex than nouns, and thus likely have more discriminative usage patterns in terms of the n-grams that they are contained in. This syntactically distinctive usage pattern then leads to lower order similarity across verbs.

However, the higher context similarity of verbs suggests that verbs typically occur with a more stable set of words than nouns do. This signals that verbs have consistent roles in syntactic constructions, leading to more dependable contextual usages, while nouns can be used in a variety of different constructions. Given the size of the effects that was found in the differences for context and order similarity across the different categories, these differences seem to reflect fundamental differences in the organization of noun and verb representations within the lexicon. This result additionally demonstrates the usefulness of the BEAGLE model of semantics (Jones 
$\&$ Mewhort, 2007) in analyzing the use of different types of distributional information in language processing, similar to past studies (Hare, et al., 2012; Johns, et al., 2018, 2020).

Sensorimotor similarity was derived from the modality/effector-specific Lancaster Sensorimotor Norms (Lynott, et al., 2020). It was found across the two experiments that the nouns produced were closer to each other in the multidimensional sensorimotor strength than verbs. Previous studies have found that the majority of English words, especially nouns, are primarily grounded in the visual modality (i.e., vision has the highest strength across all the dimensions; Lynott, \& Connell, 2009; Lynott, et al., 2020; Winter, Perlman, \& Majid, 2018). Consistently, over $80 \%$ of the noun types produced in the current study were visually dominant (see Figure 5), leading to a greater overall sensorimotor similarity across nouns. In contrast, verb types produced were more distributed across different modalities and effectors, and only $38 \%$ were primarily grounded in vision, which contributes to lower sensorimotor similarity across verbs. However, the pattern of producing verbs across various modalities and effectors may indicate that people employed an exploration-exploitation mechanism over the sensorimotor space in verb fluency, supporting the foraging model of Hills, et al. (2012).

The results of this article, along with recent trends using verbal fluency to explore theoretical issues in memory search (Abbott, et al., 2015; Hills, et al., 2012; Hills, Todd, \& Jones, 2015), demonstrate the usefulness of this data type to further develop psycholinguistic theory. Although of fundamental importance in neuropsychological and clinical domains (e.g., Taler \& Philips, 2008), verbal fluency has not received the same amount of attention as other tasks from a theoretical point of view. We showed here that by examining verbal fluency patterns, critical and consistent differences between the representations of nouns and verbs can be found. This suggests that the usage of verbal fluency tasks, when combined with computational cognitive modeling, can allow for new insights into old questions.

Although past researchers have contrasted distributional and grounded/embodied models as being competitory approaches (e.g., Glenberg \& Robertson, 2000), this article reinforces current trends in the cognitive sciences in treating these information sources as being complementary to understanding language and cognition (Bruni, Tran, \& Baroni, 2014; Johns \& Jones, 2012; Louwerse, 2008, 2011; Riordan \& Jones, 2011; Silberer \& Lapata, 2012; Wingfield $\&$ Connell, 2019). Both information types are critical to understanding lexical behaviors, and although there will be further arguments about which information stream accounts for the most 
data, examining how they interact with each other will provide important insights into how different types of statistical information combine to explain lexical behavior. 


\section{References}

Abbott, J. T., Austerweil, J. L., \& Griffiths, T. L. (2015). Random walks on semantic networks can resemble optimal foraging. Psychological Review, 122, 558-569.

Andrews, M., Vigliocco, G., \& Vinson, D. (2009). Integrating experiential and distributional data to learn semantic representations. Psychological Review, 116, 463-498.

Avery, J. E., \& Jones, M. N. (2018). Comparing models of semantic fluency: Do humans forage optimally or walk randomly? In Proceedings of the 40th Annual Meeting of the Cognitive Science Society (pp. 118-123).

Barsalou, L. W. (1999). Perceptual symbol systems. Behavioral and Brain Sciences, 22, 577660.

Barsalou, L. W. (2008). Grounded cognition. Annual Review of Psychology, 59, 617-645.

Beber, B. C., \& Chaves, M. L. (2014). The basis and applications of the action fluency and action naming tasks. Dementia \& Neuropsychologia, 8, 47-57.

Bennett, S. D., Burnett, A. N., Siakaluk, P. D., \& Pexman, P. M. (2011). Imageability and body-object interaction ratings for 599 multisyllabic nouns. Behavior Research Methods, 43, 1100-1109.

Bruni, E., Tran, N. K., \& Baroni, M. (2014). Multimodal distributional semantics. Journal of Artificial Intelligence Research, 49, 1-47.

Brysbaert, M., Mandera, P., \& Keuleers, E. (2018). The word frequency effect in word processing: An updated review. Current Directions in Psychological Science, 27, 4550.

Connell, L., \& Lynott, D. (2012). Strength of perceptual experience predicts word processing performance better than concreteness or imageability. Cognition, 125, 452-465.

Connell, L., \& Lynott, D. (2014). I see/hear what you mean: Semantic activation in visual word recognition depends on perceptual attention. Journal of Experimental Psychology: General, 143, 527-533.

Firth, J. R. (1957). A synopsis of linguistic theory, 1930-1955. In J. R. Firth (Ed.), Studies in linguistic analysis (pp. 1-32). Oxford, England: Blackwell.

Glenberg, A. M., \& Robertson, D. A. (2000). Symbol grounding and meaning: A comparison of high-dimensional and embodied theories of meaning. Journal of Memory and Language, 43, 379-401. 
Günther, F., Rinaldi, L., \& Marelli, M. (2019). Vector-space models of semantic representation from a cognitive perspective: A discussion of common misconceptions. Perspectives on Psychological Science, 14, 1006-1033.

Gureckis, T. M., Martin, J., McDonnell, J., Rich, A. S., Markant, D., Coenen, A., . . . Chan, P. (2016). Psiturk: An open-source framework for conducting replicable behavioral experiments online. Behavior Research Methods, 48, 829-842.

Hare, M. Jones, M. N., Thomson, C., Kelley, S., \& McRae, K. (2009). Activating event knowledge. Cognition, 111, 151-167.

Heard, A., Madan, C. R., Protzner, A. B., \& Pexman, P. M. (2019). Getting a grip on sensorimotor effects in lexical-semantic processing. Behavior Research Methods, 51, $1-13$.

Hills, T. T., Jones, M. N., \& Todd, P. M. (2012). Optimal foraging in semantic memory. Psychological Review, 119, 431-440.

Hills, T. T., Todd, P. M., \& Jones, M. N. (2015). Foraging in semantic fields: How we search through memory. Topics in Cognitive Science, 7, 513-534.

Johns, B. T., \& Jones, M. N. (2012). Perceptual inference through global lexical similarity. Topics in Cognitive Science, 4, 103-120.

Johns, B. T., Taler, V., Pisoni, D. B., Farlow, M. R., Hake, A. M., Kareken, D. A., . . Jones, M. N. (2018). Cognitive modeling as an interface between brain and behavior: Measuring the semantic decline in mild cognitive impairment. Canadian Journal of Experimental Psychology, 72, 117-126.

Johns, B. T. \& Jamieson, R. K. (2019). The influence of time and place on lexical behavior: A distributional analysis. Behavior Research Methods, 51, 2438-2453.

Johns, B. T. \& Dye, M. (2019). Gender bias at scale: Evidence from the usage of personal names. Behavior Research Methods, 51, 1601-1618.

Johns, B. T., Dye, M., \& Jones, M. N. (2020). Estimating the prevalence and diversity of words in written language. Quarterly Journal of Experimental Psychology, 73, 841-855.

Johns, B. T., \& Jones, M. N. (2015). Generating structure from experience: A retrieval-based model of sentence processing. Canadian Journal of Experimental Psychology, 69, 233251.

Johns, B. T., Jones, M. N., \& Mewhort, D. J. K. (2019). Using experiential optimization to build 
lexical representations. Psychonomic Bulletin \& Review, 26, 103-126.

Johns, B. T., Jamieson, R. K., Crump, M. J. C., Jones, M. N., \& Mewhort, D. J. K. (2020).

Production without rules: Using an instance memory model to exploit structure in natural language. Journal of Memory and Language.

Johns, B. T., Mewhort, D. J. K., \& Jones, M. N. (2019). The role of negative information in distributional semantic learning. Cognitive Science, 43, e12730.

Jones, M. N., Kintsch, W., \& Mewhort, D. J. K. (2006). High-dimensional semantic space accounts of priming. Journal of Memory and Language, 55, 534-552.

Jones, M. N., \& Mewhort, D. J. K. (2007). Representing word meaning and order information in a composite holographic lexicon. Psychological Review, 114, 1-37.

Jones, M. N., Hills, T. T., \& Todd, P. M. (2015). Hidden processes in structural representations: A reply to Abbott, Austerweil, and Griffiths (2015). Psychological Review, 122, 570-574.

Jones, M. N., Willits, J., \& Dennis, S. (2015). Models of semantic memory. In The oxford handbook of computational and mathematical psychology (pp. 232-254).

Koenig, Jean-Pierre (2016). Event semantics. In N. Riemer (Ed.), The Routledge Handbook of Semantics (pp. 387-402).

Landauer, T. K., \& Dumais, S. T. (1997). A solution to Plato's problem: The latent semantic analysis theory of acquisition, induction, and representation of knowledge. Psychological Review, 104, 211-240.

Lynott, D., \& Connell, L. (2009). Modality exclusivity norms for 423 object properties. Behavior Research Methods, 41, 558-564.

Lynott, D., \& Connell, L. (2013). Modality exclusivity norms for 400 nouns: The relationship between perceptual experience and surface word form. Behavior Research Methods, 45, 516-526.

Lynott, D., Connell, L., Brysbaert, M., Brand, J., \& Carney, J. (2020). The Lancaster Sensorimotor Norms: Multidimensional measures of perceptual and action strength for 40,000 English words. Behavior Research Methods, 52, 1271-1291.

Louwerse, M. M. (2008). Embodied relations are encoded in language. Psychonomic Bulletin \& Review, 15, 838-844.

Louwerse, M. M. (2011). Symbol interdependency in symbolic and embodied cognition. Topics 
in Cognitive Science, 3, 273-302.

McRae, K., Cree, G. S., Seidenberg, M. S., \& McNorgan, C. (2005). Semantic feature production norms for a large set of living and nonliving things. Behavior Research Methods, 37, 547559.

Mikolov, T., Sutskever, I., Chen, K., Corrado, G. S., \& Dean, J. (2013). Distributed representations of words and phrases and their compositionality. In Advances in Neural Information Processing Systems (pp. 3111-3119).

Moseley, R. L., \& Pulvermüller, F. (2014). Nouns, verbs, objects, actions, and abstractions: Local fMRI activity indexes semantics, not lexical categories. Brain and Language, 132, 28-42.

Murdock, B. B. (1982). A theory for the storage and retrieval of item and associative information. Psychological Review, 89, 609-626.

Murdock, B. B. (1995). Developing TODAM: Three models for serial-order information. Memory \& Cognition, 23, 631-645.

Murphy, K. J., Rich, J. B., \& Troyer, A. K. (2006). Verbal fluency patterns in amnestic mild cognitive impairment are characteristic of Alzheimer's type dementia. Journal of the International Neuropsychological Society, 12, 570-574.

Nasreddine, Z. S., Phillips, N. A., Bédirian, V., Charbonneau, S., Whitehead, V., Collin, I., ... \& Chertkow, H. (2005). The Montreal Cognitive Assessment, MoCA: A brief screening tool for mild cognitive impairment. Journal of the American Geriatrics Society, 53, 695-699.

Östberg, P., Fernaeus, S. E., Hellström, Å., Bogdanović, N., \& Wahlund, L. O. (2005). Impaired verb fluency: A sign of mild cognitive impairment. Brain and Language, 95, 273-279.

Pakhomov, S. V., Eberly, L., \& Knopman, D. (2016). Characterizing cognitive performance in a large longitudinal study of aging with computerized semantic indices of verbal fluency. Neuropsychologia, 89, 42-56.

Patra, A., Bose, A., \& Marinis, T. (2020). Performance difference in verbal fluency in bilingual and monolingual speakers. Bilingualism: Language and Cognition, 23, 204-218.

Pexman, P. M. (in press). The role of embodiment in conceptual development. Language, 


\section{Cognition and Neuroscience.}

Pexman, P. M., Muraki, E., Sidhu, D. M., Siakaluk, P. D., \& Yap, M. J. (2019). Quantifying sensorimotor experience: Body-object interaction ratings for more than 9,000 English words. Behavior Research Methods, 51, 453-466.

Piatt, A. L., Fields, J. A., Paolo, A. M., Koller, W. C., \& Tröster, A. I. (1999). Lexical, semantic, and action verbal fluency in Parkinson's disease with and without dementia. Journal of Clinical and Experimental Neuropsychology, 21, 435-443.

Plate, T. A. (1995). Holographic reduced representations. IEEE Transactions on Neural Networks, 6, 623-641.

Riordan, B., \& Jones, M. N. (2011). Redundancy in perceptual and linguistic experience: Comparing feature- based and distributional models of semantic representation. Topics in Cognitive Science, 3, 303-345.

Siakaluk, P. D., Pexman, P. M., Sears, C. R., Wilson, K., Locheed, K., \& Owen, W. J. (2008). The benefits of sensorimotor knowledge: Body-object interaction facilitates semantic processing. Cognitive Science, 32, 591-605.

Sidhu, D. M., Kwan, R., Pexman, P. M., \& Siakaluk, P. D. (2014). Effects of relative embodiment in lexical and semantic processing of verbs. Acta psychologica, 149, 3239.

Silberer, C., \& Lapata, M. (2012). Grounded models of semantic representation. In Proceedings of the 2012 Joint Conference on Empirical Methods in Natural Language Processing and Computational Natural Language Learning (pp. 14231433).

Taler, V., Johns, B. T., \& Jones, M. N. (in press). A large-scale semantic analysis of verbal fluency across the aging spectrum: Data from the Canadian Longitudinal study on Aging. The journals of gerontology. Series B, Psychological sciences and social sciences.

Taler, V., Johns, B. T., Young, K., Sheppard, C., \& Jones, M. N. (2013). A computational analysis of semantic structure in bilingual verbal fluency performance. Journal of Memory and Language, 69, 607-618.

Taler, V., \& Phillips, N. A. (2008). Language performance in Alzheimer's disease and mild cognitive impairment: a comparative review. Journal of clinical and experimental 
neuropsychology, 30, 501-556.

Taler, V., Monetta, L., Sheppard, C., \& Ohman, A. (2020). Semantic function in mild cognitive impairment. Frontiers in Psychology, 10, 3041.

Thiele, K., Quinting, J. M., \& Stenneken, P. (2016). New ways to analyze word generation performance in brain injury: A systematic review and meta-analysis of additional performance measures. Journal of Clinical and Experimental Neuropsychology, 38, 764 781.

Tillotson, S. M., Siakaluk, P. D., \& Pexman, P. M. (2008). Body-object interaction ratings for 1,618 monosyllabic nouns. Behavior Research Methods, 40, 1075-1078.

Troyer, A. K., Moscovitch, M., \& Winocur, G. (1997). Clustering and switching as two components of verbal fluency: evidence from younger and older healthy adults. Neuropsychology, 11, 138-146.

Troyer, A. K., Moscovitch, M., Winocur, G., Leach, L., \& Freedman, M. (1998). Clustering and switching on verbal fluency tests in alzheimer's and parkinson's disease. Journal of the International Neuropsychological Society, 4, 137-143.

Vigliocco, G., Vinson, D. P., Druks, J., Barber, H., \& Cappa, S. F. (2011). Nouns and verbs in the brain: a review of behavioural, electrophysiological, neuropsychological and imaging studies. Neuroscience \& Biobehavioral Reviews, $35,407-426$.

Wingfield, C., \& Connell, L. (2019). Understanding the role of linguistic distributional knowledge in cognition. PsyArXiv.

Winter, B., Perlman, M., \& Majid, A. (2018). Vision dominates in perceptual language: English sensory vocabulary is optimized for usage. Cognition, 179, 213-220. 

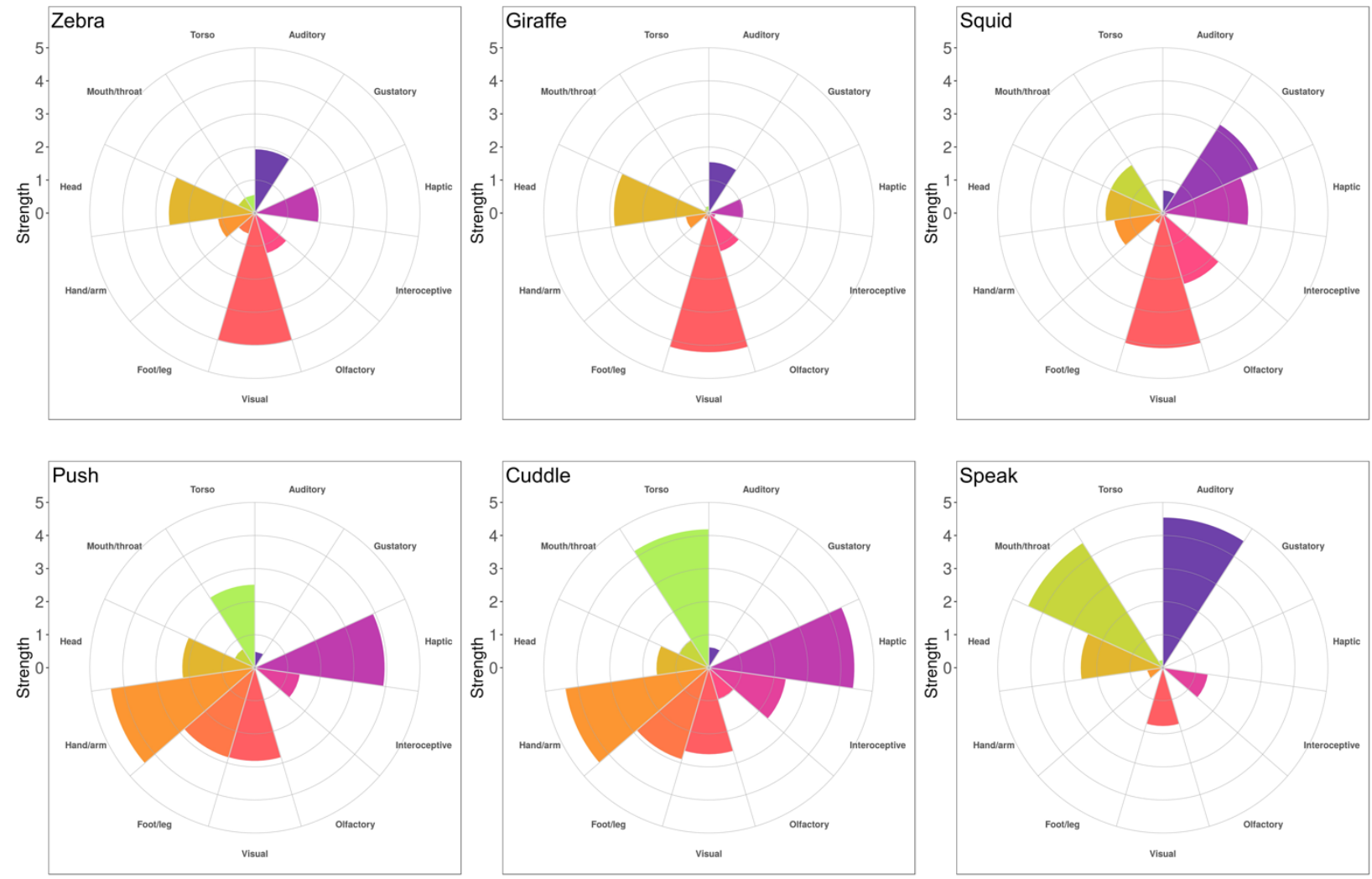

Figure 1. Polar plots of 11-dimension sensorimotor strength for selected nouns (top panels) and verbs (bottom panels). Plots were generated using the web application associated with the norms (https://www.lancaster.ac.uk/psychology/lsnorms/). 


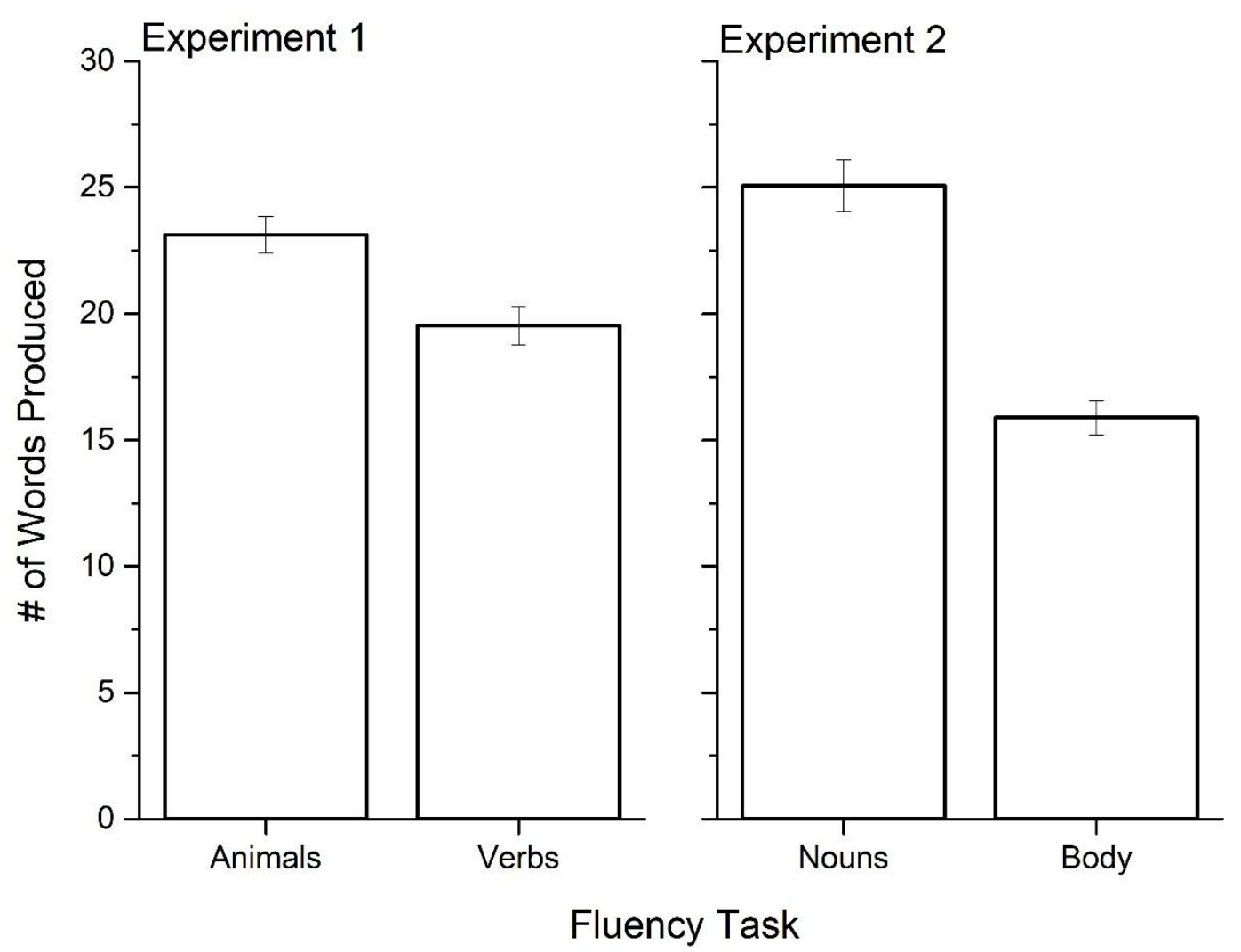

Figure 2. Number of items produced for the different verbal fluency tasks for Experiment 1 (left panel) and Experiment 2 (right panel) 


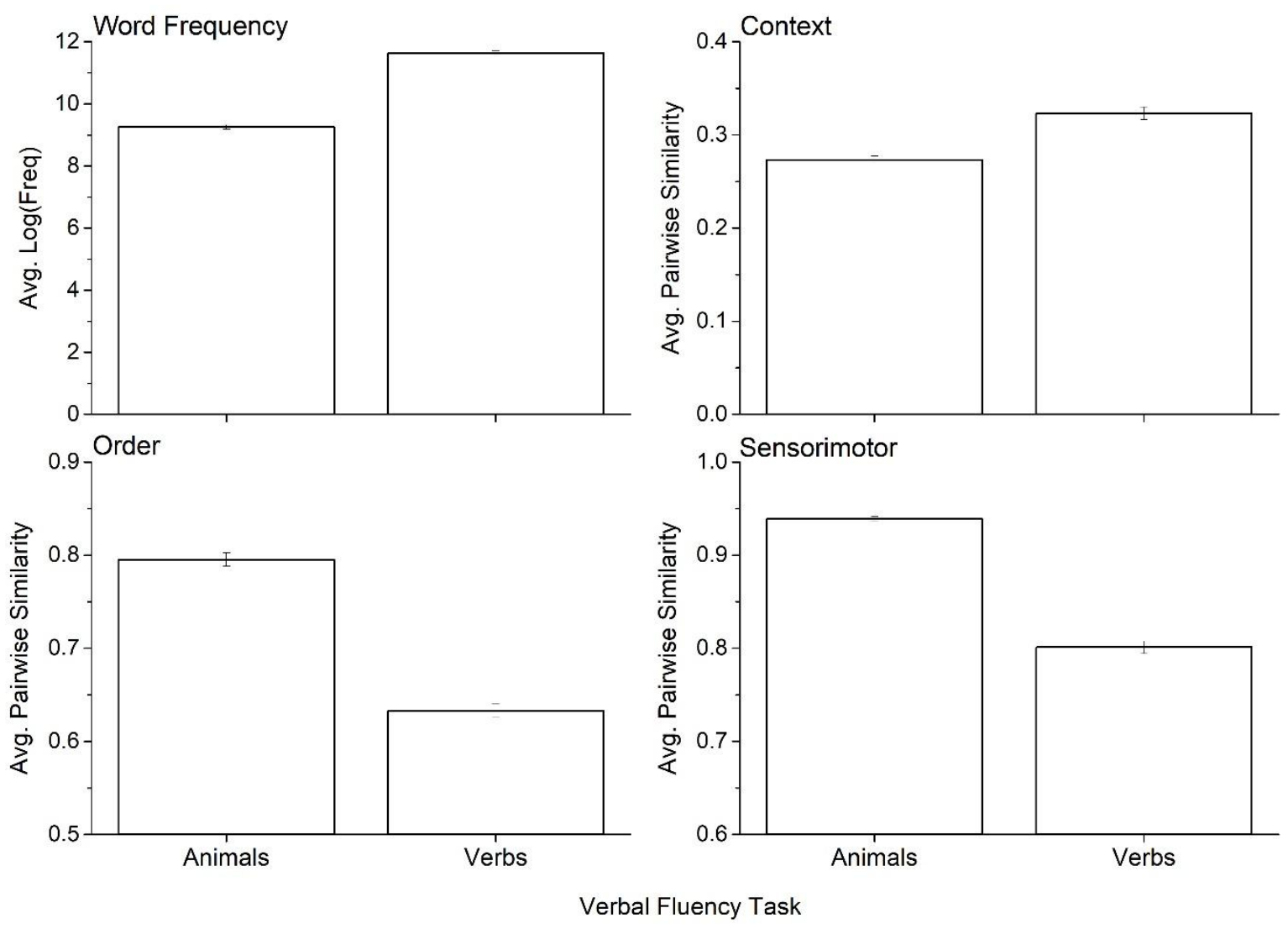

Figure 3. Values of the different information sources for animal and verb fluency. 

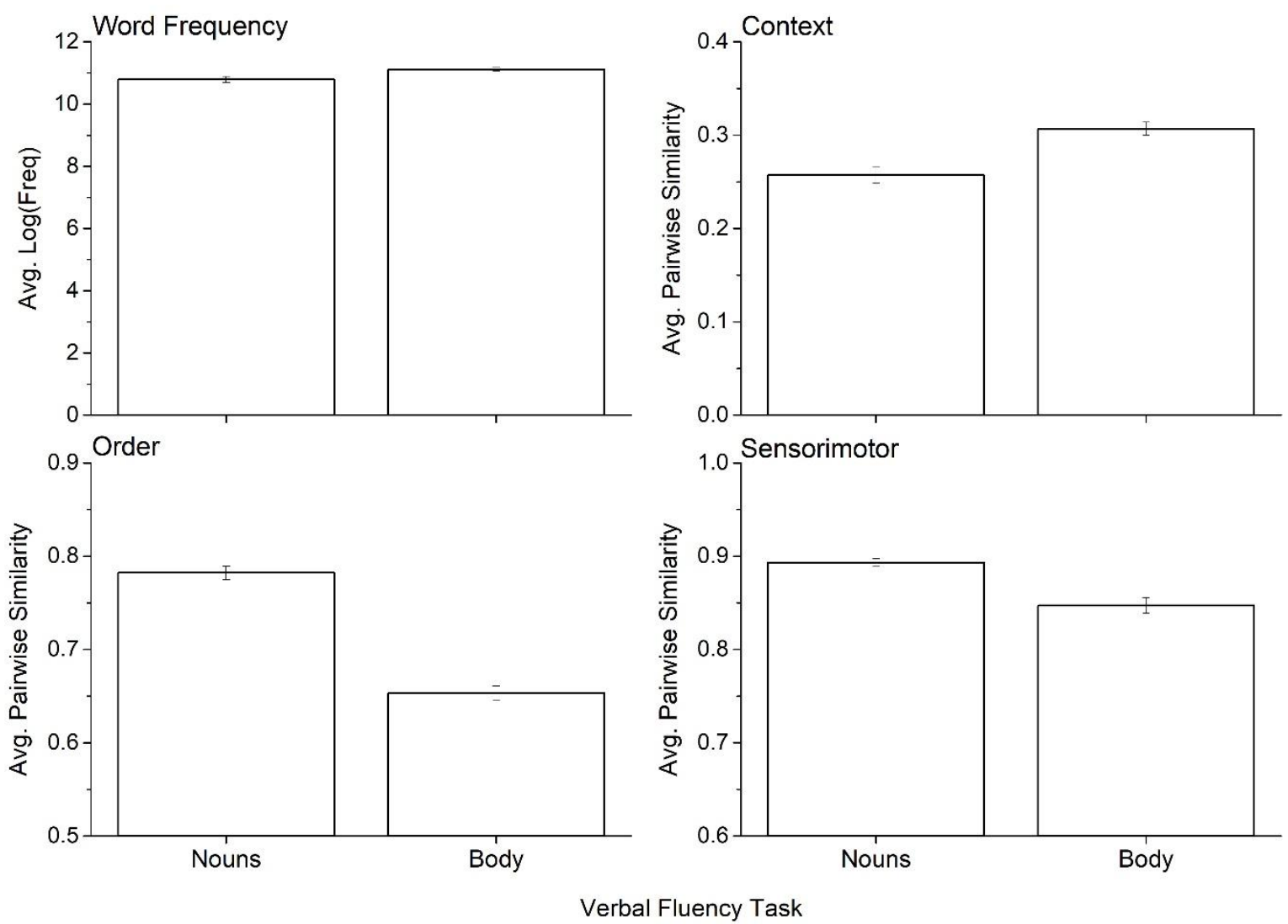

Figure 4. Values of the different information sources for noun and body movement fluency. 


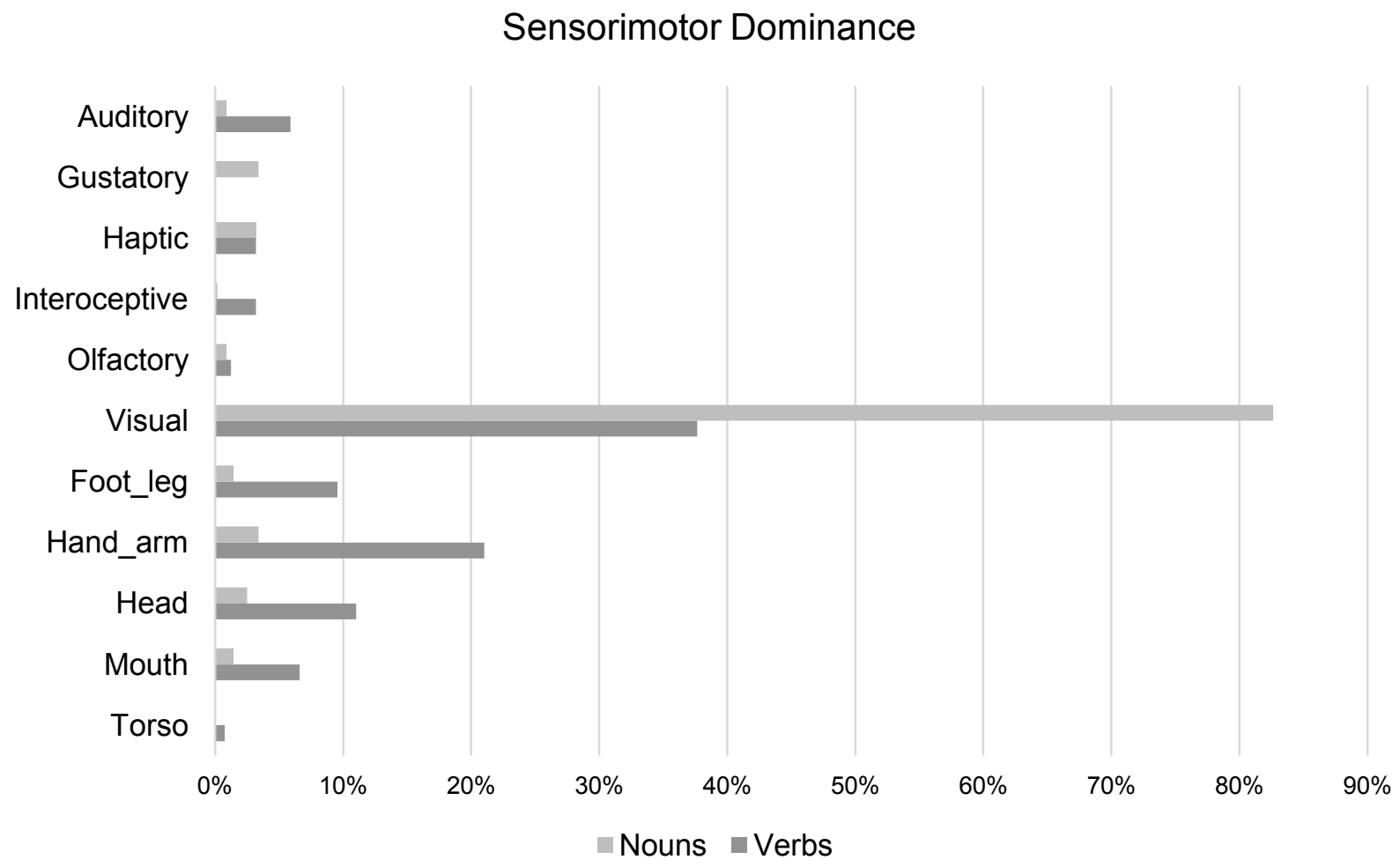

Figure 5. Percentage of noun and verb types produced in each sensorimotor category. 